Revista de Comunicación y Salud, 2020, Vol. 10, oㅡ 2, pp. 249-263

Editado por Cátedra de Comunicación y Salud

ISSN: 2173-1675

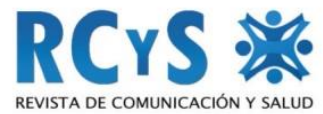

Enviado 14/08/2020

Aprobado 29/09/2020

\title{
LA CRISIS DE SALUD, DE BIENESTAR EMOCIONAL Y DE COMPETENCIAS PARA SER UN BUEN DOCENTE
}

\author{
The crisis of health and emotional and competential well-being to be a good \\ teacher
}

\author{
Marta Abanades Sánchez ${ }^{1}$ \\ Universidad Europea de Madrid. España. \\ Marta.abanades@universidadeuropea.es
}

\section{Resumen}

Han sido muchos los esfuerzos realizados por toda la comunidad docente para poder finalizar el curso académico con cierta satisfacción. La comunidad educativa ya golpeada desde hace tiempo por cuestiones de estrés y baja motivación se ve enfrentada con el paso del COVID-19 en una crisis por intentar gestionar con cierto bienestar y de manera virtual el contenido de las materias, las pruebas de evaluación, y el seguimiento de los alumnos entre otras cuestiones. Por todo esto, se ha hecho necesario que los docentes potencien sus habilidades sociales y comunicativas. En este artículo se expone la problemática sufrida por el profesorado universitario en una universidad privada en España sobre el bienestar de salud, emocional y social. Los objetivos que se plantean son los de conocer por parte de los alumnos que importancia tiene para ellos que sus profesores posean habilidades sociales y comunicativas. Además, se abordó cuáles son las características que debe tener un buen profesor. Como método de investigación se plantea un diseño descriptivo y como herramienta se utiliza un cuestionario con preguntas cuantitativas. Los resultados apuntan hacia la necesidad de que los profesores potencien sus habilidades y herramientas de comunicación, así como la escucha activa. Los docentes del siglo XXI serán aquellos donde prevalece en su persona los valores, la ética y la moral. También será necesario que dominen las competencias sociales y comunicativas, así como tener una dilatada experiencia en la profesión.

Como conclusión, los profesores que posean el conjunto de habilidades y competencias mencionados son los docentes predilectos por parte del alumnado.

Palabras clave: Competencias; Habilidades; Docentes; Bienestar; Experiencia; Alumnado.

\footnotetext{
1 Autor para correspondencia: Marta Abanades Sánchez, Universidad Europea de Madrid,
} Marta.abanades@universidadeuropea.es 
La crisis de salud, de bienestar emocional y de competencias para ser un buen docente

\begin{abstract}
Many efforts have been made by the entire teaching community to be able to finish the academic year with some satisfaction. The educational community, already hit for a long time by issues of stress and low motivation, is faced with the passage of COVID-19 in a crisis for trying to manage with certain well-being and in a virtual way the content of the subjects, the evaluation tests, the monitoring of students among other issues. For all this, it has become necessary for teachers to enhance their social and communication skills. This article presents the problems suffered by university professors at a private university in Spain regarding health, emotional and social well-being. The objectives set is to know by the students how important it is for them that their teachers have social and communication skills. In addition, the characteristics that a good teacher should have were addressed. A descriptive design is proposed as a research method and a questionnaire with quantitative questions is used as a tool. The results point to the need for teachers to enhance their communication skills and tools, as well as active listening. Teachers of the 21st century will be those where values, ethics and morals prevail in their person. It will also be necessary for them to master social and communication skills, as well as having extensive experience in the profession.

In conclusion, the teachers who possess the aforementioned set of skills and competencies are the preferred teachers of the students.
\end{abstract}

Keywords: Competences; Skills; Teachers; Well-being; Experience; Students.

Cómo citar el artículo

Abanades Sánchez, M. (2020). La crisis de salud, de bienestar emocional y de competencias para ser un buen docente. Revista de Comunicación y Salud, 10 (2), 249-263. doi: https://doi.org/10.35669/rcys.2020.10(2).249-263

\title{
1. INTRODUCCIÓN
}

\subsection{La salud y el bienestar de los docentes en los centros educativos}

Bien es conocido que la salud unida al bienestar de los docentes siempre ha sido un tema de bastante controversia. Se define salud según la Organización Mundial de la Salud (OMS) en 1946 como: "El estado completo de bienestar físico, mental y social y no solamente la ausencia de enfermedades".

Asumiendo esta definición nos hace plantearnos si en los centros educativos los educadores, docentes y maestros, han podido gozar de un equilibrio en las tres ramas, tanto físico, como mental y social.

Es cierto, que si hablamos de salud laboral hacemos referencia a estudios o acciones que tienen como finalidad conocer la importancia del trabajo en las alteraciones de la salud en una población, así como las medidas preventivas que se

Revista de Comunicación y Salud, 2020, Vol. 10, nº 2, pp. 249-263 
pueden realizar en el marco laboral (López López-Menchero, 2009). También cabe mencionar la ley 31/1995, de 8 de noviembre, de Prevención de Riesgos Laborales (BOE no 269, de 10 de noviembre de 1995) en España donde se constatan las normativas y directivas europeas relativas a la aplicación de las medidas para promover la mejora de la salud y la seguridad de los trabajadores y trabajadoras, así como el marco jurídico en el que se integra la política de prevención comunitaria. En concreto en esta ley, en el artículo 14 se recogen los derechos de protección y seguridad para los trabajadores.

Aunque lamentablemente la realidad concretamente en los centros educativos es diferente, bien es conocido los trastornos, enfermedades psicológicas, el estrés, la ansiedad, que abordan a los docentes en el ámbito educativo. Esto finalmente llega a transcender en bajas laborales temporales de los empleados.

Según Hijano (2010), el profesorado constituye un grupo muy heterogéneo. Con todo, sí hay algunas notas comunes a todo el colectivo, entre las que salta a la vista el trato con personas. Aun cuando la relación con los alumnos y alumnas es uno de los aspectos potencialmente más gratificantes, lo cierto es que en un número significativo de casos, esta comunicación está presidida por la tensión, ya sea por indisciplina de los escolares o porque el docente carece de habilidades sociales (Martínez-Otero, 2003). Este punto es totalmente trascendental y esta tomando en los últimos años gran auge entre el colectivo docente. No solo se espera del profesor que sea un excelente conocedor de la materia, sino que además tenga las habilidades sociales y de comunicación necesarias para poder transmitir el contenido a sus alumnos. Con el plan de Bolonia cada vez son más los profesores que han tenido que formarse en competencias y habilidades para de igual manera poder trasladarlas al aula.

También cabe destacar que la actividad educativa está saturada de responsabilidades (López López- Menchero, 2009). Los docentes se han convertido en parte administrativos y por otro lado agentes policiales, saturados de trabajo y requerimientos de normas que les alejan de ese profesor cuya principal responsabilidad es saber transmitir conocimientos, valores y actitudes a sus alumnos.

Actualmente se ha complicado aun más la propuesta, los profesores se vieron obligados a trasladar sus clases a sus casas y durante el final del curso académico, tuvieron que intentar continuar con sus materias animando a sus alumnos para que pudieran poner el foco de interés en la parte académica intentando dejar en un segundo plano la problemática con el COVID-19, como el impacto del aislamiento físico, el bienestar del alumno y su familia tras una perdida etc.

Todo esto ha supuesto un gran desgaste a un colectivo ya de por si saturado en funciones y responsabilidades. 
La crisis de salud, de bienestar emocional y de competencias para ser un buen docente

\subsection{La importancia de las relaciones interpersonales de los docentes.}

En este apartado vamos a poner de manifiesto la importancia que ha tomado el bienestar de los profesores para una comunicación efectiva en el aula. Dentro del perfil profesional del docente, conviene indicar que cada vez está siendo más importante para esta profesión la percepción, la comprensión y la regulación de las emociones, no solo las que tiene el propio profesional sino también las que puedan ejercer sobre los demás, en este caso, sobre su alumnado. En el ámbito educativo se encuentran tomando acciones para concienciar emocionalmente, ofreciendo cursos de formación o transmitiendo a los alumnos ciertas competencias y habilidades.

Las empresas de reclutamiento y selección de personal cada vez son más conscientes de la necesidad de profesionales con competencias socio emocionales. Buscan personas que tengan en su currículo herramientas para gestionar el talento. Volviendo al ámbito educativo, antes de vivir el colapso teniendo el $100 \%$ de las clases en sistemas virtuales en los domicilios, los profesores se caracterizaban por ser una profesión angustiosa y agobiante debido a que se encuentran interactuando de manera constante con muchas personas intentando regular sus propias emociones y la de los demás, ahora podemos hablar de una profesión agotada psicológicamente tras finalizar el trimestre en casa.

Lo que se hace presente, después de vivir junto con el COVID-19 para finalizar el curso en el ámbito educativo, es que se requieren profesionales diferentes en la enseñanza. Los docentes en el próximo curso académico necesitaran de inteligencia emocional y de una gran resiliencia y generosidad para abordar el curso académico. El docente tiene que ser un facilitador, un pedagogo eficiente, un mediador con su equipo, un docente que gestionará y diseñará sus propios recursos, conocerá el manejo de las tecnologías y sabrá utilizar los fallos como fuente de aprendizaje para desarrollar en el alumno mayor autonomía (Muñoz, 2003).

También deberán tener otro tipo de competencias como la inteligencia emocional, para poder percibir, comprender y regular emociones, tanto las propias como las de los demás (Mayer y Salovey, 1997, citado en Extremera, Fernández-Berrocal y Durán, 2003). Esta capacidad es la que permitirá a los profesores que puedan entender la suspicacia de las emociones como el manejo de estas para poder influir de manera adecuada en los procesos de enseñanza-aprendizaje de sus alumnos.

Hay diversos estudios e investigaciones sobre el estado de ansiedad y el estrés que se sufre en los entornos educativos. Dichos estudios aclaran que la labor del docente es más vulnerable que otras a sufrir enfermedades físicas y mentales (Extremera, Fernández-Berrocal y Durán, 2003).

Por todas estas razones, según Abanades (2017) se debería poder evaluar la situación de los docentes para que se sientan protegidos y a su vez competentes para desarrollar su profesión con resultados de éxito, controlando las situaciones, gestionando de manera óptima las relaciones dentro de su organización con padres,

Revista de Comunicación y Salud, 2020, Vol. 10, oㅡ 2, pp. 249-263 
La crisis de salud, de bienestar emocional y de competencias para ser un buen docente

compañeros y alumnos y, finalmente, orientando de manera triunfante a sus alumnos mediante un desarrollo de actividades más práctico y menos teórico.

En el ámbito educativo, también las relaciones interpersonales constituyen un componente básico de las diversas competencias (Zabalza, 2003). La extendida idea de que enseñar es transmitir conocimientos resulta insuficiente para reflejar la riqueza y variedad de los intercambios que se producen en la relación profesor-alumno. La enseñanza establece procesos de intercambio que van mucho más allá de la información.

Bradford comenta que la enseñanza consiste básicamente en la relación interpersonal del encuentro:

El proceso de enseñar-aprender es una transacción humana que une al maestro, al estudiante y al grupo en su conjunto de interacciones dinámicas que sirven de marco a un aprendizaje entendido como cambio que se incorpora al proyecto vital de cada individuo. El objetivo básico de la educación es el cambio y crecimiento o maduración del individuo; esto es, una meta más profunda y compleja que el mero crecimiento intelectual. (Bradford, 1973, pág. 21).

Las relaciones interpersonales que se mantienen en la enseñanza presentan una serie de retos por parte del docente, algunos de ellos los describimos a continuación:

1. Cuanto mayor sea el porcentaje de alumnos dentro del aula mayor complicación será la gestión para el docente. Según Feldman en 1976, la importancia de la calidad de la enseñanza radica en tener la capacidad de saber relacionarse con los alumnos. El tener relaciones interpersonales satisfactorias será cualidad ecuánime que además valoraran los propios alumnos en sus profesores.

2. El propio estilo de liderazgo que tenga el docente, algunas de las aportaciones más conocidas son las investigaciones que realizaron Lewin, Lippit y White, 1939, que describieron tres diferentes estilos de liderazgo instructivo:

a) El autoritario o directivo, donde el docente especifica las actividades y cómo llevarlas a cabo, ordena lo que se debe realizar en cada caso para alcanzar los objetivos y reparte los cometidos individuales.

b) Un segundo estilo sería el del líder democrático o participativo, donde los alumnos se sienten más protagonistas de su aprendizaje y participan y colaboran en los proyectos y en la toma de decisiones de las actividades. Este líder también se involucra y, en vez de realizar críticas personales, reconoce el trabajo realizado.

c) Y, por último, cabe destacar el líder laissez faire o pasivo. En este caso, el docente tiene un estilo pasivo, solo reacciona cuando se lo solicitan el resto de las personas.

De otras investigaciones determinaron el perfil de los docentes dominantes. Las actuaciones de estos profesionales se basan en controlar todas las actividades siempre a través de órdenes y mandatos, donde priman las amenazas y constantes reproches sin valorar el trabajo o esfuerzo de los alumnos (Anderson y Brewer, 1945).

Revista de Comunicación y Salud, 2020, Vol. 10, nº 2, pp. 249-263 
Seguramente de todas estas clasificaciones el docente se encuentra identificado con varias acciones y actitudes de varios modelos. Lo ideal sería que hubiera un equilibrio entre el docente y el alumno.

Como señala McDermott, existen formas directivas de comunicación en clase que tienen éxito y otras que no la tienen, de la misma manera que existen formas autoritarias exitosas y otras que no lo son:

Ningún estilo pedagógico es intrínsecamente mejor que otro. Si alumnos y profesores pueden entender el estilo lo suficientemente bien como para construir sus planes de trabajo y una relación de confianza, entonces la relación funcionará. (McDermott, 1977, pág. 101).

3. Para finalizar el último gran reto que se le presenta al docente sería el clima del aula. Investigadores coinciden que es fundamental para poder desarrollar el método de enseñanza-aprendizaje en los alumnos que exista un buen clima en el aula, este concepto abarca muchas opiniones. Fox lo define de la siguiente manera:

El clima de una institución educativa resulta del tipo de programa, de los procesos utilizados, de las condiciones ambientales que la caracterizan como una institución y como un agrupamiento de alumnos, de los departamentos, de la persona, de los miembros de la dirección. Cada institución posee un clima propio y distinto. El clima determina la calidad de vida y la productividad de los profesores y alumnos. El clima es un factor crítico para la salud y para la eficacia de una escuela. Para los seres humanos, el clima puede convertirse en un factor de desarrollo. (Fox, 1980: pág. 457).

En definitiva, saber relacionarse con los alumnos, el propio estilo de liderazgo que establezcamos con ellos y el clima que generemos en el aula, ya sea en un espacio presencial o a través de los seminarios virtuales, será fundamental para tener las capacidades de establecer relaciones interpersonales en el ámbito educativo con calidad.

\subsection{Ser un buen docente antes los nuevos retos educativos}

Es comprensible que los docentes necesiten de un buen currículo de contenidos, y sobre todo experiencias que puedan avalar el trabajo realizado hasta el momento. $\mathrm{Y}$, aun así, todo esto por si solo tampoco marcara la diferencia en ser o no un buen docente.

Dentro del ámbito educativo, ser un buen docente es también una cuestión personal, ya que, al ser una rama vocacional, la persona se prepara durante el recorrido de toda su vida, de manera constante y continua y esto no es un trabajo fácil. Hasta el momento, hemos tenido en consideración el bienestar y la salud del profesor además de las interacciones interpersonales con la comunidad educativa (alumnos, profesores, padres, jefes y responsables...) para desarrollar una labor de éxito. 
El cambio de ser un docente a ser otro mejor, es una cuestión también de actuar bien, en función de nuestra experiencia. Si ser docente no es solo un logro personal, transformarse en un docente mejor es un proceso social y personal (Knight, 2005). Por eso, además de las habilidades interpersonales y de las competencias, no podemos obviar la experiencia, esa parte práctica de la labor del docente. La clase no debería centrarse en la instrucción directa. No gusta a ninguno y nadie se siente especialmente motivado. A los profesores tampoco les gusta, se sienten como si estuvieran disparando información al vacío. Los seres humanos no deberían ser pasivos. Cuando se juntan, deberían interaccionar, resolver problemas o elaborar cosas (Robinson, 2015).

A continuación, para poder abordar la cuestión de la experiencia por parte del docente, vamos a describir algunos de los consejos por Knight (2005) sobre que pueden hacer los profesores para facilitar la experiencia en el trabajo.

1. Intentar tener la perspectiva de que trabajar muchas horas no es más que una señal de incompetencia y no de un signo de distinción. Aunque Campbell y Neill, en el 1994, confirmaron que las horas que trabajan los docentes británicos no universitarios sería una prueba de una deficiente práctica profesional.

2. A los docentes se les exige gran cantidad de horas y ciertamente el trabajo de despacho es menos satisfactorio que cuando nos encontramos interactuando dentro del aula. Saber distribuirse el tiempo no es tarea fácil. Leer artículos, atender el correo, evaluar, redactar comentarios, preparar las sesiones y por supuesto poder reflexionar, sobre todo, es una tarea complicada, necesitaremos ser eficaces.

3. Este autor declara la necesidad de poder reflexionar con mucha frecuencia. Poder escribir se hace más sencillo cuando sabemos lo que queremos decir, por eso se hace necesario poder reflexionar con mayor asiduidad. De acuerdo con el hallazgo de que los nuevos profesores universitarios que tienen éxito son los que escriben algo a diario, se puede escribir lo que se ha reflexionado mientras se pasea por el despacho (Boice, 1992).

4. Solicitar que nos hagan comentarios sobre los primeros borradores de nuestros materiales de enseñanza, en vez de hacerlo cuando los productos están prácticamente terminados.

5. Como muchas de las tareas en el ámbito educativo, tenemos la manía de recoger datos (reuniones, evaluaciones etc.). ¿Se hace necesario conversarlo?, reflexionemos si es necesario tener que mantenerlo.

6. Intentemos aprender técnicas de respuesta inmediata, es decir, poder despachar con agilidad los correos electrónicos.

7. Concienciémonos de cuánto tiempo merece la pena dedicarle a una reunión y abandonémosla cuando se cumpla el plazo.

8. Hay que aprender a decir que "no".

9. Que los estudiantes puedan aprender unos de otros y ha facilitarse la información para nosotros es ahorrar tiempo. Estas prácticas además son buenas para el aprendizaje de los alumnos.

10. Leer mucho. Se hace necesario leer cosas que puedan ayudarnos a revisar nuestra investigación, nuestra enseñanza y nuestro trabajo. 
La crisis de salud, de bienestar emocional y de competencias para ser un buen docente

11. Se asocia siempre el poder tener una buena forma física y un bienestar mental. Tal y como hemos abordado con anterioridad, podremos ver nuestro trabajo de manera diferente si mantenemos un equilibrio en nuestro bienestar físico y mental.

12. Para intentar que las energías mentales vayan en nuestro beneficio y no al contrario, necesitaremos en ocasiones de trabajo de autoayuda, limpieza emocional y programación neurolingüística.

13. Buscar oportunidades de hacer cosas donde se valore y aprecie nuestra vitalidad personal y profesional.

14. Parece que lo normal no es que reconozcamos los éxitos de los demás, para las instituciones casi la valía o no de cada uno queda definida por los fracasos y no por los éxitos. Hay que celebrar las cosas que nos salen bien y saber reconocer a los demás el trabajo bien hecho.

Siguiendo estas premisas, se intenta que el colectivo docente pueda prepararse para tener bienestar de salud, mental, emocional y que pueda realizar con éxito sus clases implementando al máximo su capacidad comunicativa y social. Además, ser eficientes en nuestro trabajo nos ayudara a mantener el equilibrio y tener más tiempo para aquellas cuestiones de interés.

\section{OBJETIVOS}

Tras abordar el escenario en el que vive el ámbito docente, nos planteamos algunas propuestas relacionadas con la importancia que le da el alumno a ciertas habilidades y competencias, así como a la metodología que utiliza. Los docentes en su mayoría ya son conscientes de lo que pueden hacer para mejorar el equilibrio entre el bienestar de salud mental y emocional, la calidad de las relaciones y el desarrollo de habilidades comunicativas, por eso se hace necesario también poner el foco de atención en los alumnos para conocer la importancia de estas.

Como objetivos nos proponemos las siguientes cuestiones:

- Conocer la importancia que le da el alumnado a las habilidades comunicativas del docente.

- Analizar cuáles son las características más demandadas por los alumnos para ser un buen profesor.

\section{METODOLOGÍA}

La propuesta metodológica de investigación es de tipo descriptivo con un enfoque cuantitativo. El instrumento utilizado es un cuestionario creado en Google/forms desde donde se le trasladaron a los alumnos algunas cuestiones sobre la importancia que dan a las competencias y a la marca personal de sus docentes.

Aunque en este estudio no vamos a desarrollar todas las cuestiones, las preguntas de este cuestionario fueron extraídas de los autores Arévalo, Sáenz, \& González (2016)

Revista de Comunicación y Salud, 2020, Vol. 10, oㅡ 2, pp. 249-263 
en su artículo "La marca personal como elemento decisorio para un buen maestro". Estos autores buscan identificar las cualidades que tiene un buen maestro. Según confirman cada vez son más los centros en el ámbito educativo que buscan a profesionales de calidad que se encuentren reconocidos y que tengan una buena marca personal.

\subsection{Muestra}

La muestra elegida son 115 alumnos de diferentes materias y titulaciones de Grado y Postgrado. Se envió el enlace para la realización del cuestionario y se obtuvieron 75 resultados. Las titulaciones y materias que conforman a este grupo son:

- Grado en Marketing: 1ำ curso materia: Competencias I Eficacia personal y profesional.

- Grado en Marketing: 2o curso materia: Habilidades de Comunicación Oral y Escrita.

- Máster universitario en Marketing y Comunicación: Modulo Comunicación y Marca personal.

- Máster universitario en Recursos Humanos: Modulo Entrevista Personal.

A continuación, en la siguiente gráfica podemos ver el tanto porcentual de los alumnos que colaboraron: Grado con 43 respuestas y postgrado con 32 respuestas.

\section{TIPO DE FORMACION}

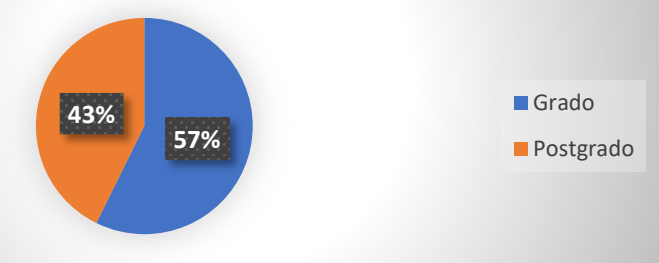

Figura 1. Muestra de alumnos.

Fuente. Elaboración propia.

Consideramos que al pasar el cuestionario al final del semestre, y con todo lo sucedido en el último trimestre tras el COVID-19, hemos obtenido un menor número de respuestas de las que esperábamos. 


\section{DISCUSIÓN}

Como hemos comentado no nos vamos a centrar en todas las preguntas del cuestionario, pero si en aquellas que tienen especial relevancia con nuestra propuesta de investigación.

En este artículo hemos comentado la importancia del bienestar de salud en el docente y de esta manera poder transmitir unos valores y unos principios en sus materias. También hemos abordado la importancia de la comunicación y competencia comunicativa en el docente como así no podemos obviar la relevancia de la experiencia adquirida en su profesión.

En la siguiente figura se exponen tres ámbitos de actuación, uno personal donde los valores y la ética está implícita en el docente, otro ámbito sería el de la formación que abarcaría no solo los estudios sino también la experiencia que tienen los profesores y por último el ámbito docente que abarcaría las habilidades sociales y comunicativas que tiene el docente. A los alumnos se les comenta que especifiquen cada una de las cuestiones puntuando de 1 a 3 , siendo un 1 el más importante para ellos y con un 3 el que menos.

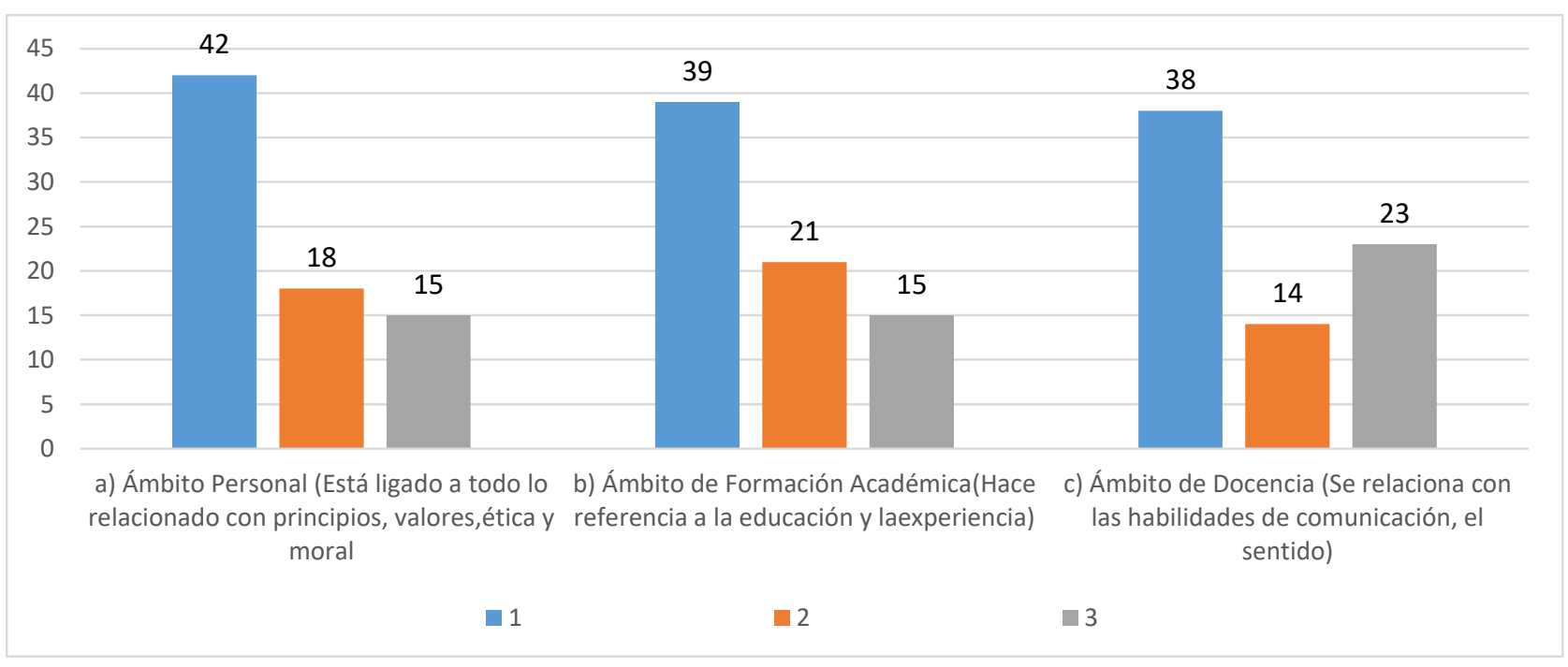

Figura 2. La importancia de los ámbitos en el docente.

Fuente: Elaboración propia.

Como vemos en los tres ámbitos de actuación, los alumnos confirman la importancia que tiene para ellos que sus profesores posean y transmitan unos valores y principios, también que tengan práctica y experiencia en su trabajo además de que puedan manifestar y desarrollar habilidades de comunicación.

En el ámbito personal el $56 \%$ de los alumnos confirmaron su importancia adjudicándole un 1. En el ámbito de formación académica, el $52 \%$ de los alumnos 
confirmaron la opción 1. Y en último ámbito relacionado con la docencia y las habilidades de comunicación, confirmaron el 50\% alumnos. Estos datos se ajustan teniendo en consideración que los alumnos seleccionaron cada una de las tres respuestas en función de su grado de importancia.

Otra de las cuestiones que hemos abordado en el artículo es la capacidad y habilidad para ser un buen docente. Se les preguntó a los alumnos por cuales serían aquellas características que podrían definir a un buen profesor en función de las siguientes competencias: organización, puntualidad, capacidad de escucha, capacidad para transmitir conocimientos, autoridad en el aula y la retroalimentación de las evaluaciones. En la siguiente figura vemos las respuestas.

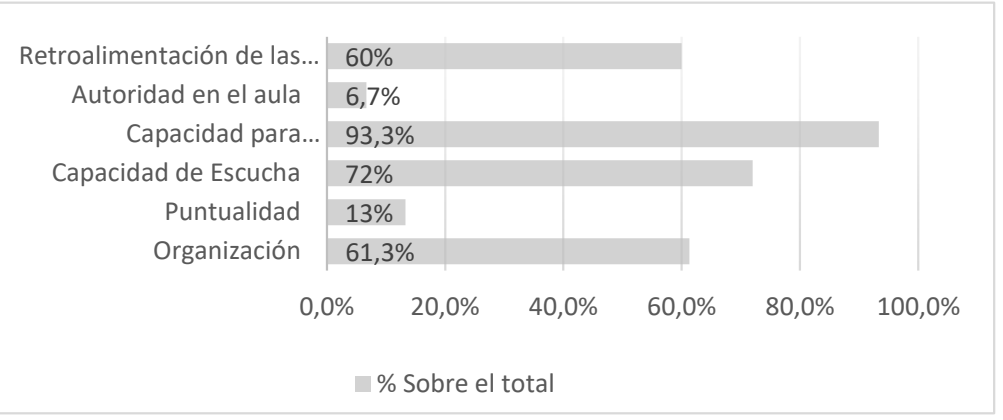

Figura 3. Características que describen a un buen docente.

Fuente: Elaboración propia.

Como podemos apreciar en los resultados aquellas características más importantes que podrían definir a un buen profesor serian la capacidad de transmitir conocimientos, con un total porcentual del casi el $94 \%$, detrás de esta encontramos la capacidad de escuchar, con un total porcentual del $72 \%$, y en tercera posición, la organización, con un total del casi del $62 \%$. Aunque con menos importancia, han valorado la retroalimentación de las evaluaciones con un total del $60 \%$, la puntualidad con un $13 \%$ y la autoridad en el aula con casi un $7 \%$.

\section{CONCLUSIONES}

Tras las respuestas obtenidas en función de los resultados llegamos a las siguientes conclusiones. Si bien diversos autores coinciden en la importancia del bienestar del docente para poder transmitir de manera efectiva unos contenidos, unas competencias y una ética en cada una de sus materias. En verdad el colectivo docente esta pasando por una crisis, ya que es un sector que ya venia con cierto desgaste en su profesión 
debido a la interacción constante con la comunidad educativa, así como por la cantidad de horas y burocracia administrativa que tiene que abordar.

Otra de las cuestiones es que además en el último trimestre, el profesor se ha visto obligado a realizar sus clases de manera virtual, donde se le ha exigido una mayor interacción, comunicación y atención del rendimiento de sus alumnos, así como a la asistencia y participación de estos. Como sabemos, la competencia tecnológica está más que implantada en nuestra sociedad, pero los docentes, en su mayoría, siguen teniendo ciertas deferencias en su manejo como así la preferencia de sesiones presenciales antes que virtuales.

En cuestión de ámbitos de actuación, más de la mitad de los alumnos coinciden en la importancia que tiene que sus profesores se manejen en los ámbitos de actuación, así como el saber transmitir valores y tener una ética. Además, coinciden en que posean habilidades sociales y comunicativas, así como una experiencia dilatada en su profesión.

Por los resultados obtenidos y en relación con la introducción, más de la mitad de los alumnos se posicionan en tener profesores que posean ese equilibrio mental, social y emocional para poder acercarse a lo que ellos determinan ser buenos profesionales de la docencia.

Otro de los apartados que hemos argumentado en nuestra introducción ha sido el cómo ser un buen docente. Si bien cuando les preguntamos a los alumnos sobre las características que tiene que poseer un buen docente confirman la importancia de profesores con capacidades y habilidades sociales y comunicativas, aquellos que además manejen técnicas de comunicación como la escucha activa.

Si consideramos los objetivos propuestos llegamos a las siguientes conclusiones.

Nuestro primer objetivo consistía:

- Conocer la importancia que le da el alumnado a las habilidades comunicativas del docente.

Tanto autores como Zabalza (2003), Knight (2005) entre otros confirman la necesidad por parte del docente de las habilidades sociales y comunicativas. Ya no es plus como podíamos pensar hace años, sino una necesidad por parte de todos los profesionales en el ámbito educativo el poder mostrar en los alumnos empatía y escucha activa para también transmitirles y enseñarles competencias y herramientas de comunicación.

Por su parte los alumnos, según las respuestas obtenidas valoran significativamente que el profesor posea habilidades de comunicación, escucha activa entre otras que aparentemente no le dan tanta importancia como a la puntualidad y a la autoridad dentro del aula.

El otro de los objetivos que habíamos planteado es: 
La crisis de salud, de bienestar emocional y de competencias para ser un buen docente

- Analizar cuáles son las características más demandadas por los alumnos para ser un buen profesor.

Si ser docente no es solo un logro personal, transformarse en un docente mejor es un proceso social y personal (Knight, 2005). Las habilidades interpersonales, las competencias y la experiencia son elementos esenciales en la mochila del docente.

Los alumnos valoran la experiencia que tienen los profesores, así como sus habilidades emocionales, comunicativas y sociales, pero como pudimos observar en las respuestas de los alumnos, valoran aún más el ámbito personal del docente. Aquel que es capaz de transmitir unos valores y una ética, que comprende, no juzga y predomina su moral ante las cuestiones y situaciones a tratar. Todos estos elementos contribuyen al logro de las competencias profesionales de los estudiantes, como el aprendizaje cooperativo, las claves en la enseñanza de las TIC, etc.

Otro de los puntos a destacar es la necesidad de la formación del docente en las TIC. Si observamos las figuras 2 y 3 , se corrobora que los alumnos quieren docentes que destaquen en su ámbito personal y que sean grandes transmisores de contenidos. Esta premisa unida a la nueva era en la enseñanza-aprendizaje de manera virtual, hará que los docentes trabajen más en herramientas y competencias digitales para impartir las clases de manera virtual con una buena base en las TIC.

Como conclusión podemos destacar que se hace necesario que el profesor posea habilidades y competencias comunicativas, así como herramientas de comunicación y, preferiblemente, que posea una dilatada experiencia. También se le avalará la capacidad de mantener relaciones interpersonales de éxito. Podríamos considerar que estas capacidades podrían proporcionar a los docentes ese equilibrio que necesitan de bienestar social y emocional. Además, se prevé seguir manteniendo en el escenario universitario un modelo híbrido, donde conjuguen clases presenciales y también virtuales. Los profesores que tengan la posibilidad de crear empatía, saber escuchar y posean manejo en la competencia digital, serán elegidos y denominados por sus alumnos como buenos docentes. En el plano de bienestar y salud, además de los consejos para ser eficaces en el trabajo, realizar ejercicio entre otras, los docentes necesitaran ser resilientes y desarrollar inteligencia emocional para poder seguir sobrellevando todas las acciones laborales con una actitud positiva.

\section{RECOMENDACIONES}

Tras las respuestas obtenidas se considera que en futuras investigaciones se hace necesario poder incluir en la muestra alumnos de otras facultades y escuelas y si fuera posible una muestra de universidades representativa a nivel nacional e internacional.

También se hace considerable pasar la muestra en momentos menos críticos del curso académico, mejor al inicio o durante el mismo para poder obtener mayor número de respuestas y no finalizando el curso académico.

Revista de Comunicación y Salud, 2020, Vol. 10, nº 2, pp. 249-263 
La crisis de salud, de bienestar emocional y de competencias para ser un buen docente

\section{REFERENCIAS}

Abanades Sánchez, M. (2017). La inteligencia emocional en docentes de educación superior. Estudio de caso sobre su influencia en los programas de la Universidad Europea de Madrid: evaluación y líneas de actuación (Doctoral dissertation).

Anderson, H. H., \& Brewer, H. M. (1945). Dominative and Socially Integrative Behavior of Kindergarten Teachers. Applied Psychology Monographs, (6), 9.

Arévalo, C. C., Sáenz, S. S., \& González, A. A. (2016). La marca personal como elemento decisorio de un buen maestro. Libre Empresa, 13(2), 73-84. doi: 10.18041/libemp.2016.v13n2.26205

Boice, R. (1992). Lessons learned about mentoring. New directions for teaching and learning, 50, 51-61. doi: 10.1002/tl.37219925007

Bradford, L. P. (1973). La transacción enseñar-aprender. La Educación Hoy, 21-27.

Campbell, R. J., \& Neill, S. R. S. J. (1994). Primary teachers at work. Psychology Press.

Feldman, K. A. (1976). The superior college teacher from the students' view. Research in Higher Education, 5(3), 243-288. doi: 10.1007/BF00991967

Fernández Berrocal, P., Extremera, N. y Ramos, N. (2004). Validity and reliability of the Spanish Modified Version of the Trait Meta-Mood Scale. Psychological Reports, 94, 751-755. doi: $10.2466 /$ pr0.94.3.751-755

Fox, R. F. (1980). Treatment of writing apprehension and its effects on composition. Research in the Teaching of English, 14(1), 39-49. https://www.jstor.org/stable/40171027

Hijano, L. M. M. (2010). La importancia de la salud laboral docente: estudio de un caso concreto. espiral. Cuadernos del profesorado, 3(5), 11-29. doi: $\underline{10.25115 / e c p . v 3 i 5.900}$

Knight, P.T. (2005). El profesorado de Educación Superior. Formación para la excelencia. Ed. Narcea.

Ley 31/1995, de 8 de noviembre, de Prevención de Riesgos Laborales. Jefatura del Estado. (BOE oㅡ 269, de 10 de noviembre de 1995). Madrid.

Lewin, K., Lippitt, R., \& White, R. K. (1939). Patterns of aggressive behavior in experimentally created "social climates". The Journal of social psychology, 10(2), 269-299. doi: $\underline{10.1080 / 00224545.1939 .9713366}$

López Menchero, J. L. (2009). La salud laboral de los docentes. Recuperado de http://www.educaweb.com/noticia/2009/03/26/salud-laboraldocentes 13542.html. 
La crisis de salud, de bienestar emocional y de competencias para ser un buen docente

Martínez-Otero, V. (2003): La salud mental del profesorado, en Comunidad Escolar, 713. Recuperado el 15 de enero, 2018 de la siguiente dirección electrónica: http://comunidad-escolar.pntic.mec.es/713/tribuna.html

McDermott, R. P. (1977). Kids make sense: An ethnographic account of the interactional management of success and failure in one first-grade classroom. Department of Anthropology, Stanford University.

Muñoz, R. F. (2003). Competencias profesionales del docente en la sociedad del siglo XXI. In Organización y gestión educativa: Revista del Fórum Europeo de Administradores de la Educación (Vol. 11, No. 1, pp. 4-7). Ciss Praxis.

Robinson, K (2015). Escuelas Creativas. Barcelona. Ed. Grijalbo.

Salovey, P., \& Mayer, J. D. (1990). Emotional intelligence. Imagination, cognition and personality, 9(3), 185-211. doi: 10.2190/DUGG-P24E-52WK-6CDG

Salovey, P., y Sluyter, D. J. (1997). Emotional Development and Emotional Intelligence. Educational Implications. Nueva York: Basic Books.

Salovey, P., Mayer, J. D., Goldman, S. L., Turvey, C., \& Palfai, T. P. (1995). Emotional attention, clarity, and repair: Exploring emotional intelligence using the Trait MetaMood Scale. Emotion, disclosure, and health,125-154. doi: 10.1037/10182-006

Zabalza, M.A. (2003). Competencias docentes del profesorado universitario. Calidad y desarrollo profesional. Ed. Narcea.

Zabalza, M.A. (2007). La enseñanza universitaria. El escenario y sus protagonistas.

Ed. Narcea.

\section{AUTORA}

\section{Marta Abanades Sánchez}

Doctora en Educación por la Universidad Europea de Madrid. Licenciada en Psicopedagogía y Diplomada en Educación Social. Cuenta con varios programas de postgrado: Máster Internacional en Coach Profesional y Ejecutivo, Máster en Recursos Humanos y Máster Universitario en Formación al Profesorado. Profesora de materias como habilidades sociales, habilidades directivas, competencias y liderazgo. Ha asumido diferentes cargos como directora de programas de postgrado, coordinadora en materias como TFG y prácticas en el área de Educación. Cuenta con varias colaboraciones en proyectos de investigación y actualmente IP del proyecto interno: "Ecologías de aprendizaje en el Siglo XXI. Competencias sociales y tecnológicas necesarias en el ámbito laboral".

Líneas de investigación: habilidades y competencias, inteligencia emocional y formación del profesorado.

Orcid ID: https://orcid.org/0000-0002-4303-6332

Google Scholar: https://scholar.google.es/citations?hl=es\&user=yiyFbFcAAAAJ

Resear Gate: https://www.researchgate.net/profile/Marta Abanades 